\title{
THE ROBOTS WITH SOUL AND THE USE OF THE ONTOLOGIES IN THE HUMAN-ROBOT COMMUNICATION
}

\author{
Elena Mogoș, Luminița Dumitriu \\ Department of Computer and Information Technology, „Dunarea de \\ Jos" University of Galati, Romania \\ (email: Elena.Mogos@ugal.ro, Luminita.Dumitriu@ugal.ro )
}

\begin{abstract}
Robots with soul become an important field of robotics and have several issues. An interesting issue is their ability to perceive human emotions differently as the human brain. In order to recognize the human emotions a robot with soul needs to focus on Artificial Intelligence and an Artificial Neural Network, recognizing the words, the languages and the specific patterns and identifying the context and the user's intention. In this work we propose a robot with soul, which uses an emotional ontology - based Artificial Neural Network, created in Matlab 2019 , offering to the humans more privacy, a personalized conversation and a new emotion's achievement.
\end{abstract}

Keywords: the robots with soul, Artificial Intelligence, Artificial Neural Network, ontologies.

\section{THE ROBOTS WITH SOUL}

A robot with ,soul" is a humanoid robot that can engage in the human-like conversations. A robot with ,soul" is built on Machine Learning/Deep learning algorithms and on a model of Artificial Neural Network and Artificial Intelligence software. In the conversations with a human a robot with "soul" uses a user audio interface and different text, the human emotions and the human voice. A robot with "soul" communicates with a human through the dialogue, recognizes the keywords and the human expressions, understands the context of a speech, deciphers the human emotions and can offer a quasihuman experience.

A humanoid robot is a robot designed to be similar to a human. It has the same basic physical structure and some capabilities of a human. In the future it will resemble people. In this work we present two robots with "soul", Pepper and Sophia and according to them we create our own robot with "soul" that uses the emotion ontology in the communication with a human.

\section{THE ROBOTS WITH SOUL IN THE HUMAN ROBOT COMMUNICATION}

The robots with "soul" had been developed a lot in the last decades, but they have only proven useful in the laboratories. Nowadays there are robots with "soul" not expensive and more effective.

\subsection{Pepper}

Pepper is the first robot with "soul" in the world that can read the human emotions (Fig. 1). He was designed by Softbank Robotics as a personal assistant who observes the environment and acts according to the environment. Pepper is able to recognize the human gestures, the verbal expressions and he can analyze the tone of the human voice to understand his emotions, which allow him to communicate with human in a natural way, including through some "graceful" gestures (https://www.softbankrobotics.com/emea/en/pepper). 


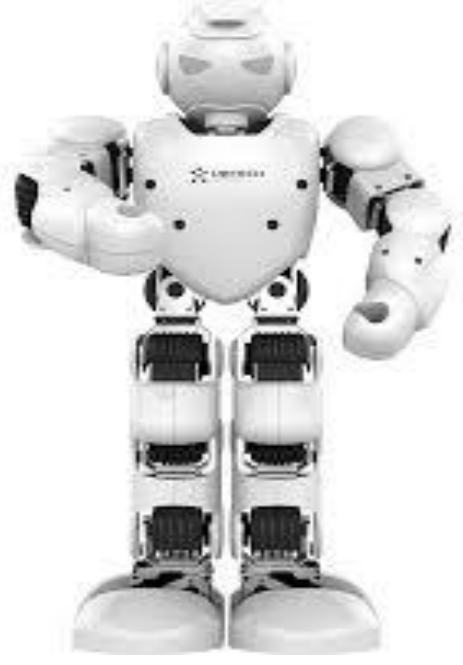

Fig.1. Pepper - the first robot with ,soul” in the world

(https://logicalisdigitallyspeaking.com/informationinsights-iot/interview-pepper-the-humanoid-robotunveils-ai-thoughts/)

The humans can interact with Pepper using the touch screen of the robot. Pepper can communicate with a human by showing the images and the animations on the screen of his tablet. Also Pepper is equipped with the motors localized in his wrist, elbow, hand and shoulder which allow him to move in any position as a human and can estimate the human emotions and human level of attention. The Pepper's module AL Autonomous Life makes the robot to have "soul" and helps AL Mood module to decipher the human emotions. Despite of these characteristics, Pepper is limited to only few emotions of the full spectrum of emotions that a human can feel because he has a low processing power. He must use an external device Raspberry $\mathrm{Pi}$ to receive/send the additional information that makes him "feel" (Fig.2).

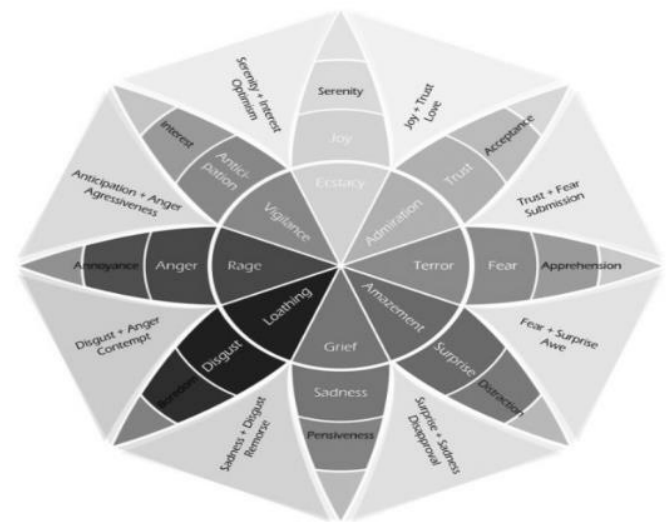

Fig.2. The deciphered emotions of Pepper (Rozanska and Podpora, 2019)

\subsection{Sophia}

Sophia is a robot with "soul" created by Hanson Robotics, she has an artificial intelligence, visual data processing software and facial recognition software. Sophia can imitate the human facial gestures and the human expressions and she is able to answer to some certain questions as some predefined topics (she can speak about the weather). In the communication with a human, Sophia uses a voice recognition software developed by Alphabet Inc., Google's parents company and she is designed to become smarter in time. She includes AI software, created by Singularity NET which analyzes the human conversation and extracts the data that allow her to improve the future responses in the humanrobot speech. Sophia is the first robot with "soul" which received the citizenship of Saudi Arabia and she is used in the nursing homes to help the old people (Fig.3).

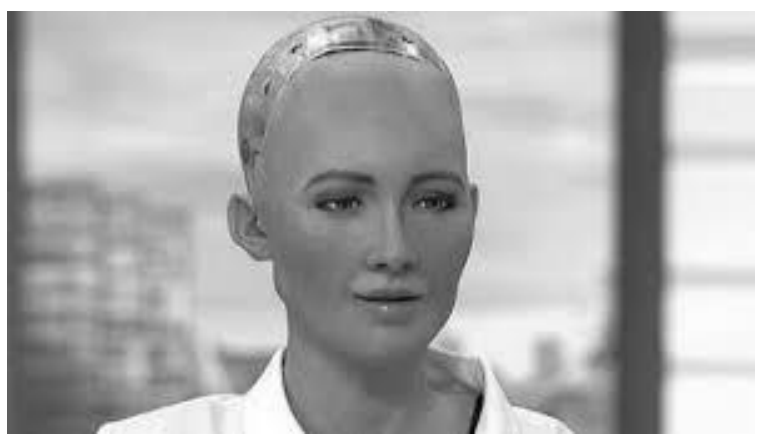

Fig.3. Sophia - the first robot with ,soul” with the citizenship of Saudi Arabia

(https://ro.wikipedia.org/wiki/Sophia_(robot))

\section{ONTOLOGIES}

The ontology is a formal representation of a multitude of the concepts from the field of artificial intelligence and the relationships between these concepts. The ontology contains a hierarchical description of the most important concepts in artificial intelligence, the properties of these concepts (attribute-value) in order to give them an appropriate type.

The ontologies are grouped in some different languages based on:

- predicate logic (OCML-Open Configuration and Management Layer, Fuzzy-logic, CYCL);

- on the Web (RDF-Resource Description Framework, SHOE);

- OWL (Web Ontology Language). 


\subsection{The ontologies in the human - robot} communication

The ontology in the human-robot communication is a set of the knowledge terms, including the vocabulary, the semantic interconnections and some simple rules of the inference and the logic for some particular topics (Fig.4).

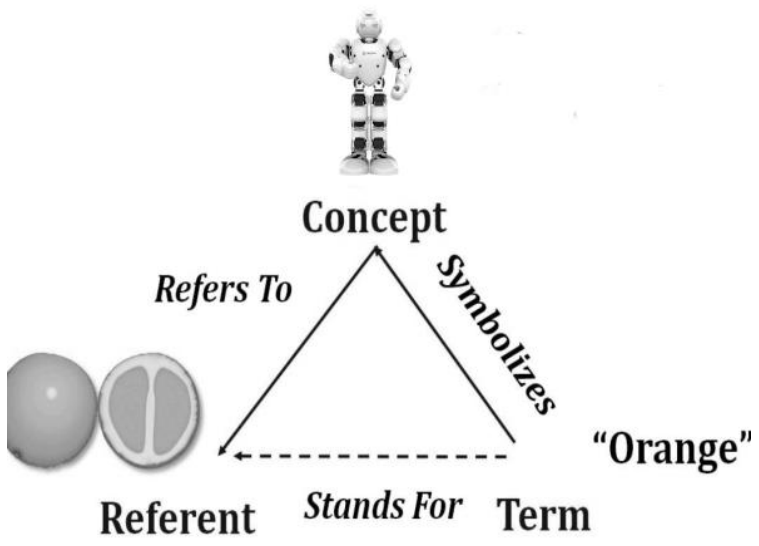

Fig.4. An ontology in the human-robot communication (https://profs.info.uaic.ro/ busaco/teach/courses/wad e/presentations/web09SemanticWeb-OntologiiOWL.pdf)

\subsection{The emotion ontologies in the human-robot communication}

The emotion ontology is the ontology of the affective phenomena such as the emotions, the moods, the appraisals and the subjective feelings, designed to support the human-robot speech. The emotion ontologies cannot be only some scientific terms with a precise definition and include some popular builders. The researchers from the field of the artificial intelligence have the difficult task to discover what a humanoid robot means when he appeals an emotion from the human speech and which the mechanism of his message transmission is. For a humanoid robot it is difficult to express the emotion in one language. In the world there are many languages and each language has different constructions for each emotion, some extremely complicated as a message, as well as the aspect of understanding it.

In our work we propose a classification of the human emotion in the form of the emotion ontology, created in Matlab R2019 (Fig. 5). The proposed ontology has 8 main nodes for each main emotion and each node has 2 or more secondary nodes. The emotion which shows the surprise has two secondary nodes: shocked and astonished.

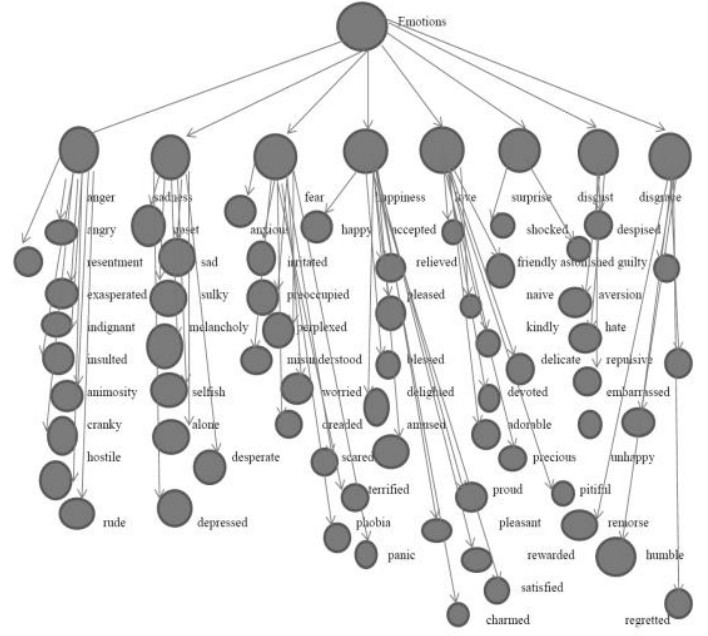

Fig.5. Proposed ontology in the human-robot communication

The main emotions from our work are: anger, sadness, fear, happiness, love, surprise, disgust and disgrace. The secondary emotions for the main emotions of the proposed emotion ontology are:

- anger: angry, resentment, exasperated, indignant, insulted, animosity, cranky, hostile, rude;

- sadness: upset, sad, sulky, melancholy, selfish, alone, desperate, depressed;

- fear: anxious, irritated, preoccupied, perplexed, misunderstood, worried, dreaded, scared, terrified, phobia, panic;

- happiness: happy, relieved, pleased, blessed, delighted, amused, proud, pleasant, rewarded, satisfied, and charmed;

- love: accepted, friendly, naive, kindly, delicate, devoted, adorable, precious, pitiful;

- $\quad$ surprise: socked, astonished;

- disgust: despised, aversion, hate, repulsive;

- disgrace: guilty, embarrassed, unhappy, remorse, humble, regretted.

\section{THE ROBOTS WITH ,SOUL” AND THE EMOTION ONTOLOGIES IN THE HUMAN ROBOT COMMUNICATION}

In the human-robot communication the robots with "soul" use the emotional ontologies to act and be "alive" as a human. In our work we proposed a robot with "soul" which uses both the proposed emotional ontology and the spectrum of light. For each main emotion from above there is a main color. For each secondary emotion there is a shade of color. We can see it in Fig. 6 and Fig. 7. 


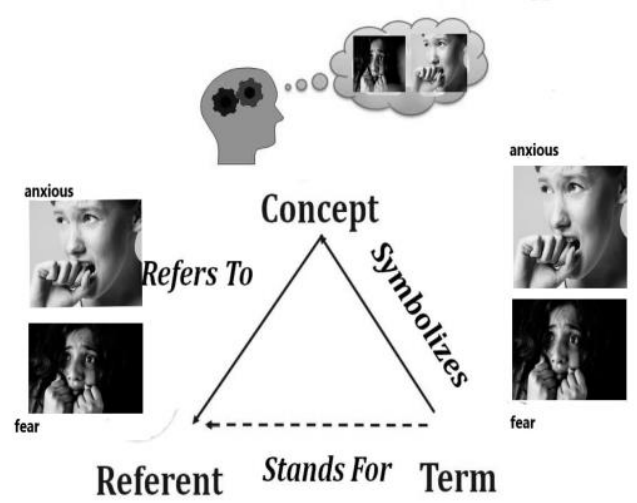

Fig.6. The emotions in the human brain

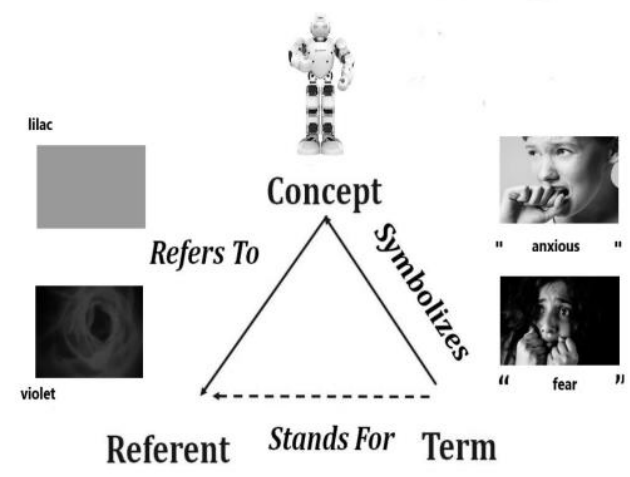

Fig.7. The emotions in the human-robot communication

4.1. The representation of the information in the human-robot communication

In the human-robot communication our emotions are recognized by software based on the fuzzy sets (Fig. $8)$. The fuzzy sets contain the color frequency $(\mathrm{Hz})$ and each main emotion (Fig. 9). Each color represents an emotion and it has the same color frequency and the same wavelength (for example, the happiness is represented by yellow).

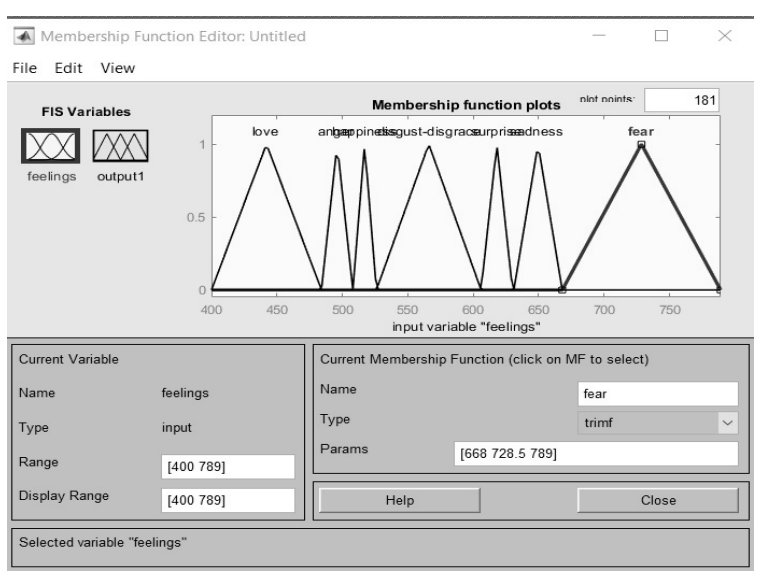

Fig.8.The fuzzy sets with the human emotions

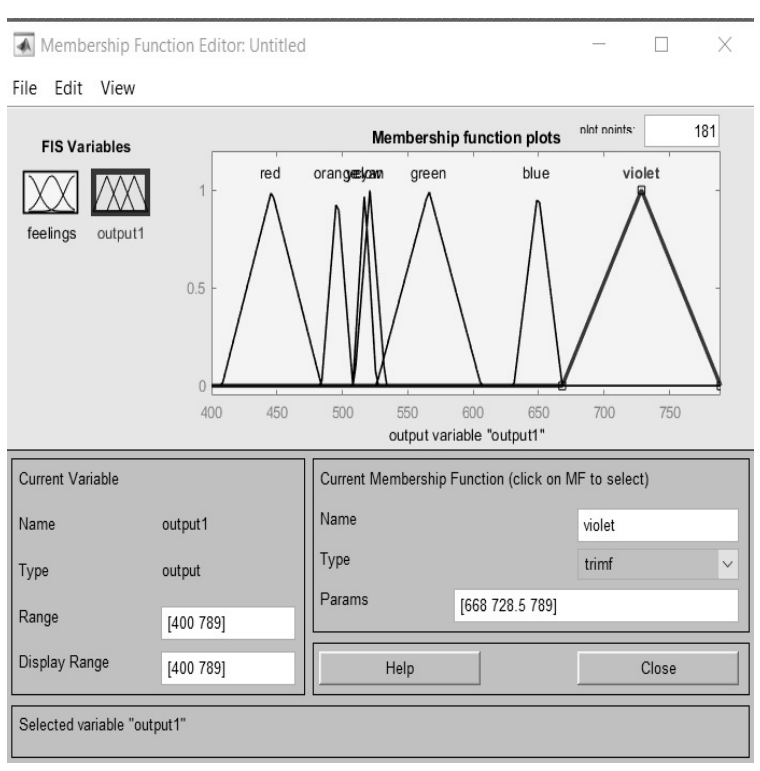

Fig.9. The fuzzy sets with the color frequency for each color.

The colors frequency in $\mathrm{Hz}$ used in the human-robot communication is represented in Table 1.

Table 1 The color's frequency used in the humanrobot communication

\begin{tabular}{|c|c|c|}
\hline Color & Frequency & Wavelength \\
\hline Violet & $668-789 \mathrm{~Hz}$ & $380-450 \mathrm{~nm}$ \\
\hline Blue & $631-668 \mathrm{~Hz}$ & $450-475 \mathrm{~nm}$ \\
\hline Cyan & $606-630 \mathrm{~Hz}$ & $476-495 \mathrm{~nm}$ \\
\hline Green & $526-606 \mathrm{~Hz}$ & $495-570 \mathrm{~nm}$ \\
\hline Yellow & $508-526 \mathrm{~Hz}$ & $570-590 \mathrm{~nm}$ \\
\hline Orange & $484-508 \mathrm{~Hz}$ & $590-620 \mathrm{~nm}$ \\
\hline Red & $400-484 \mathrm{~Hz}$ & $620-750 \mathrm{~nm}$ \\
\hline
\end{tabular}

The fuzzy rules of our software are presented in Fig. 10.

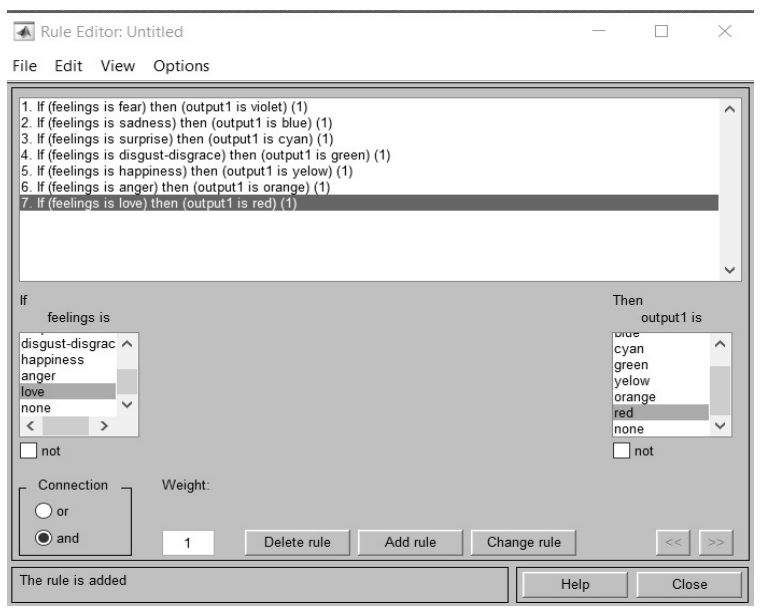

Fig.10.The fuzzy rules of the our proposed software used in human-robot communication 
Our proposed emotions depend on how the colors of the light spectrum are perceived by the human and the robot with "soul" when they communicate each other. The humans describe the colors that they perceived using the secondary emotions from our proposed ontology. The robot with ,soul" uses the color's brightness, shade, tint or tone and whether it's cool or warm toned to express his emotions. Red reflects the feeling of love. The robot with ,soul” uses red to stimulate the human body and the human mind and to increase human blood circulation. Orange reflects the feeling of anger. The robot with „soul" uses orange to heal the human lungs and to increase the human energy levels. Yellow reflects the feeling of happiness. The robot with ,soul" uses yellow to stimulate the nerves and the purity of the human body. Green reflects the feeling of disgustdisgrace. The robot with "soul" uses green to relieve the human stress and to help him to heal. Cyan reflects the feeling of surprise. The robot with "soul" uses cyan to help the human to be euphoric and to be full of energy. Blue reflects the feeling of sadness. The robot with "soul" uses blue to soothe the human illnesses and to treat the human pain. Violet reflects the feeling of fear. The robot with "soul" uses violet to alleviate human fear.

\subsection{The processing of the information in the human- robot communication}

The frequency of every audio signal perceived by a humanoid robot in the communication with a human is analyzed and compared with the spectrum of the light.Every color from the spectrum of the light is associated with the fuzzy sets, the colors (Fig.8). The samples of the audio signal from human - humanoid robot communication with the duration of 2-3 seconds are processed and a new spectrum of the frequencies is obtained. The new spectrum of the frequencies is analyzed and is associated with the fuzzy sets, the human emotions (Fig. 9). The human emotions are represented in our proposed ontology and the words in English from our ontology are used to compare the result of the humanoid robot speech recognition from the same samples of the audio signal with our fuzzy sets, the human emotions. If the comparison had a result, the humanoid robot identifies a secondary human emotion and chooses to alter the color of the main emotion to the specific shade of the secondary emotion, otherwise the humanoid robot chooses the color of the main emotion from our proposed ontology. The humanoid robot uses the fuzzy rules to transform the obtained colors for the human emotions in an audio signal which it is sent back to the human (Fig.10).

\section{CONCLUSIONS}

The era of the robots with "soul" is started and numerous robots with "soul" are used in the applications developed to assist the humans and the animals. There exist numerous challenges to the humans in the competing with the robots with "soul" and the humans are trying hard to match these types of the robots in speed, perfection and durability as a human. With such performances the robots with "soul" are better and smarter than the humans and can surpass the human in any field. Robots with "soul" can be made highly tolerant, more beauty and more versatile and can be the "mirror image" of a human.

\section{REFERENCES}

Rozanska, A. and M. Podpora (2019). Multimodal sentiment analysis applied to interaction between patients and a humanoid robot, Science. Direct, Volume no. 52, Issue 27, pp. 411-414;

https://logicalisdigitallyspeaking.com/informationinsights-iot/interview-pepper-the-humanoid-robotunveils-ai-thoughts);

https://www.softbankrobotics.com/emea/en/pepper; https://ro.wikipedia.org/wiki/Sophia_(robot));

https://profs.info.uaic.ro/ busaco/teach/courses/wade /presentations/web09SemanticWeb-Ontologii-OWL. Pdf. 